\title{
A systematic overview of cancer immunotherapy: an emerging therapy
}

\begin{abstract}
Radiation, surgery and chemotherapy constitute the "traditional weapons" to fight cancer. But these treatment strategies aim at killing fast proliferating cancer cells and are often associated with many side-effects due to a lack of specificity resulting in normal cells being destroyed. The consequences of such tools are high morbidity leading to patient discomfort and in some scenarios, even death. Thus, the use of such tools is limited and the approach of targeted delivery systems is being exploited currently. Targeted Immunotherapy represents a potential and innovative means to combat cancer. It has become one of the popular means of anticancer therapies due to relatively fewer toxic effects compared to traditional chemotherapy and thereby offers better patient compliance. Cancer vaccines designed against a specific tumor antigen have been efficiently utilized to trigger immune responses against tumor cells. This review provides sheds light on the history of immunotherapy, brief introduction to the concept of immunotherapy and the different types (active and passive), key players of the immune system involved in the anti-tumor defense activities, concept of "immunesurveillance escape", role of tumor antigens in the design of cancer vaccines, past and the on-going research in the field of immunotherapy based cancer vaccines, and lastly the limitations of cancer vaccines.
\end{abstract}

Keywords: cancer immunotherapy, tumor antigen, $\mathrm{cd} 4^{+} \mathrm{t}$ cells, $\mathrm{cd} 8^{+} \mathrm{t}$ cells, dendritic cells (DCS), passive immunotherapy, monoclonal antibodies, cytokines, active immunotherapy, cancer vaccine
Volume 5 Issue 2 - 2017

\author{
Rutika Kokate \\ Sidney Kimmel Cancer Center,Thomas Jefferson University, \\ USA
}

Correspondence: Rutika Kokate, Sidney Kimmel Cance Center,Thomas Jefferson University, USA, Tel 6825522799 Email rutika.kokate@jefferson.edu

Received: January 01, 2017 | Published: February 21, 2017
Abbreviations: ADCC, antibody dependent cell mediated cytotoxicity; CDC, complement dependent cytotoxicity; CTL, cytotoxic t lymphocyte; DC, dendritic cell; GM-CSF, granulocyte-macrophage colony stimulating factor; HPV, human papilloma virus; HBV, hepatitis b virus; IL-2, interleukin 2; IL-12, interleukin 12; IFN, interferon; MHC, major histocompatibility complex; MAB, monoclonal antibody; NK, natural killer cell; TCR, t cell receptor; TSA, tumor specific antigen; TSA, tumor associated antigen; VLP, virus like particle

\section{Introduction}

Cancer is one of most common fatal diseases afflicting people globally. It is believed that cancer will become the leading cause of death across the US population in the near future, thus increasing the need for novel cancer therapeutics. ${ }^{1}$ Cancer is a complex heterogeneous disease characterized by uncontrolled/unregulated division of cells. ${ }^{2}$ Despite tremendous progress in the field of cancer biology, cancer remains one of the most deadly diseases to treat. ${ }^{3}$ The genetic and phenotypic complexity of this disease underlies its diversity and resistance to therapeutics. Conventional cancer treatment strategies, including radiation, surgery and chemotherapy, play a significant role in the treatment of primary tumors. However, cancer recurrence due to metastasis remains a significant challenge. The inherent ability of the immune defense system to recognize and destroy cancer cells is believed to hold promise toward the eradication of cancers. Researchers are therefore aggressively seeking novel ways to exploit immune function for cancer prevention. Recently, the approach of targeted immune-based therapies is being exploited. Moreover, the success of the monoclonal antibody Herceptin (Transtuzumab) has revolutionized this field. ${ }^{4}$ Immunotherapy represents a potential and innovative means to combat cancer. Targeted immunotherapy has become one of the popular means of anticancer therapies due to better patient compliance. Because of encouraging results obtained from clinical trials, research aimed towards developing a "cancer vaccine" is being carried out extensively. ${ }^{5}$ This mini-review focuses on the history, basic concept, key immune players, the current ongoing research, hurdles and future aspects in the field of targeted immunotherapy against cancer.

\section{Immune system}

The immune "army" is comprised of immune cells such as the eosinophils, macrophages, natural killer (NK) cells and lymphocytes ( $\mathrm{T}$ and $\mathrm{B}$ cells). Amongst all these players, the $\mathrm{T}$ and $\mathrm{B}$ lymphocytes primarily mediate specific targeted antitumor response. ${ }^{6}$ The $\mathrm{CD} 4^{+} \mathrm{T}$ cells act as "helper cells" and secrete specific cytokines depending upon their profile either Th1 or Th2. The Th1 $\mathrm{CD}^{+} \mathrm{T}$ cells mainly secrete IFN- $\gamma$ and IL2.IFN- $\gamma$ is important for the activation of macrophages and microglial cells and facilitate enhanced phagocytic activity. IL2 also known as the T cell growth factor promotes the differentiation of $\mathrm{T}$ cells into effector (cytotoxic) $\mathrm{T}$ cells and aids in the development of memory $\mathrm{CD} 8^{+} \mathrm{T}$ cells thus ensuring a robust secondary immune response. ${ }^{7}$ The $\mathrm{Th} 2 \mathrm{CD}^{+} \mathrm{T}$ cells mainly secrete IL4, IL5 and IL13 which help in maturation and growth of B cells to plasma cells that actively secrete antibodies and are also involved in Antibody Dependent Cell mediated Cytotoxicity (ADCC). ${ }^{7}$ Cytotoxic $\mathrm{CD}^{+} \mathrm{T}$ Lymphocytes or CTLs are the major effector $\mathrm{T}$ lymphocytes that are tumoricidal and secrete specific cytokines (Figure 1) ${ }^{8,9}$ They are MHC (Major Histocompatibility Complex) class I restricted while the $\mathrm{CD}^{+}$helper $\mathrm{T}$ lymphocytes are MHC class II restricted. The 
MHC class restriction adds specificity to the function of T cells as it allows the T cell receptor (TCR) to bind to the respective MHCpeptide (antigen) bound complex on the host cells infected with viral proteins or pathogens. $\mathrm{CD}^{+} \mathrm{T}$ cells being MHC I restricted bind class I MHCs expressed on most cells in the body while $\mathrm{CD}^{+} \mathrm{T}$ cells bind to class II MHCs expressed on specialized antigen presenting cells (APCs). ${ }^{10}$ APCs such as macrophages and dendritic cells (DC) are also important. The role of macrophages in developing antitumor effects is a bit controversial and depends largely on their phenotype, M1 or M2. ${ }^{11,12}$ M1 macrophages are tumor suppressive while M2 are tumor promoting. ${ }^{12}$ DCs are considered as "professional APCs" as they efficiently capture and process the antigenic peptide $(\mathrm{Ag})$ and display it via MHC class I and MHC class II presentation so that it can be easily recognized by $\mathrm{CD} 8^{+}$and $\mathrm{CD} 4^{+} \mathrm{T}$ cells respectively (Figure 2)..$^{13,14}$

\section{Cancer immunotherapy}

William B. Coley, considered a pioneer in the field of cancer, introduced the concept of immunotherapy by identifying the potential of immune cells in treating cancers and designed a crude vaccine known as 'Coley's Toxin' consisting of killed bacteria that was administered to cancer patients. The patients presented with fever and chills but complete remission of their cancer in some cases. ${ }^{15,16}$ His experiment served as a stepping stone for future cancer immunotherapy. ${ }^{17}$ Progress toward advances in cancer immunotherapy however, was not without initial setbacks. A significant hurdle was attributed to the difficulty of in vitro culturing immune cells, particularly lymphocytes in vitro. In 1976, interlukin-2 (IL-2), previously known as the T cell growth factor was cloned and was noted for its ability to support the growth of T lymphocytes ex vivo. Studies thereafter, could examine the role of T lymphocytes against cancer cells, leading to the first characterization of a cancer antigen in $1991 .^{18}$ Based on this and other studies, it became widely accepted that the immune system could detect cancer cells and kill them. ${ }^{19}$ Such observations also led to the concept that cancer cells express specific proteins (tumor antigens) on their surface which may be specific or over expressed in cancer cells and may not be found or scarcely expressed in normal cells.

\section{Tumor antigens}

Tumor antigens are primarily characterized as two major types. ${ }^{20}$ Tumor Specific Antigens (TSAs) are unique to cancer cells and are not expressed on normal cells. TSAs may arise due to point mutations in genes. They may be unique to an individual or may be expressed in various tumors but not normal tissues. The second major type of tumor-antigens is termed Tumor Associated Antigens (TAAs). TAAs are expressed by both tumor and normal cells. They are not tumor specific and their use may result in autoimmunity against normal tissue expressing that Antigen. Examples include MAGE, GAGE, and NYESO1. ${ }^{21}$ Despite the ability of our immune system to recognize unique antigens expressed by tumor cells, they also can avoid immune attack by employing several "tricks" termed as "tumor immune escape". For example, the tumor microenvironment, consisting of masses of tumor cells and surrounding stroma, forms a barrier thus limiting immune cell invasion of the primary tumor. In addition, cancer cells may secrete immunosuppressive substances that may inhibit the immune response. ${ }^{22,23}$ Furthermore, the immune system may consider cancer cells as "self" since they are of the same origin expressing very similar antigens. Most noted is the ability to express self-antigen in the context of the Major Histocompatibility Class I (MHC I) complex expressed on the surface of tumor cells that is recognized by host immune cells as a non-threatening signal. Thus, the body's immune system behaves as if it is "blind" to cancer cells. This phenomenon is known as "tumor tolerance". There is also a possibility that the tumor cells expressing TSAs or TAAs, with MHC I are down-regulated in their surface expression. Thus, allowing the tumor cell to hide from the immune system by not displaying the antigenic peptide and thus is not recognized by the immune system. ${ }^{24}$ Together, these mechanisms of tumor escape make it difficult for the hosts to initiate an optimal anti-tumor immune response. To recognize the full potential of immunotherapy, it will be necessary to develop novel approaches to elevate and/or complement immune-based vaccines.

To this end, the goal of cancer immunotherapy must be:

A) To specifically target cancer cells;

B) To recruit efficient immune cells that can generate a robust and long lasting response; and

C) Most importantly prevent relapse. ${ }^{19}$

\section{Types of cancer immunotherapy}

Cancer immunotherapy may involve passive or active immunotherapy alone or in combination with conventional cancer treatments.

\section{Passive immunotherapy}

Passive immunotherapy does not rely on the body's natural immune system to attack cancer cells but uses components of the immune system such as antibodies which can be made in the laboratory to target tumor antigens. ${ }^{24}$ Some examples include Herceptin, Avastin, Rituxan, Campath, Zevalin. ${ }^{21}$ Monoclonal Antibodies (mAb) are the most widely used form of targeted cancer immunotherapy in clinic today. ${ }^{25} \mathrm{mAbs}$ bind to a specific target (antigen) on the tumor cell and their mode of action depends on their ability to engage with growth receptors or with pro-apoptotic targets inducing apoptosis of cancer cells. mAbs also facilitate antigen presentation. As discussed before, DCs are the major APCs of the immune system and play pivotal role in priming of tumor specific $\mathrm{T}$ cells. They express a variety of cell surface receptors and are capable of binding and internalizing Ag-Ab complexes, which eventually lead to their activation and maturation. Mature DCs present the antigenic peptide via MHC Class I or MHC Class II peptide complexes and thus can launch a $\mathrm{T}$ cell mediated response (Figure 1) (Figure 2) ${ }^{26}$ Herceptin and Cetuximab are examples of such mAbs that bind to HER2 and EGFR receptor respectively. These mAbs not only block the growth signaling cascade but also facilitate antigen presentation through formation of immune complexes that induce a potent $\mathrm{T}$ cell response. It was seen that the patients who received Herceptin treatment generated effective $\mathrm{T}$ cell responses. Additionally, mAbs may activate components of the immune system via Fc portion based interactions eventually promoting antibody-dependent cell-mediated cytotoxicity (ADCC) and complement-dependent cytotoxicity (CDC) by macrophages and NK cells. ${ }^{21}$ Herceptin is known to mediate ADCC via NK cells and monocytes. ${ }^{26}$ Likewise, cetuximab, the EGFR antibody also helps in DC priming and promotes antitumor responses. In vitro studies indicate that cetuximab augments DC opsonozation of cancer cells and also helps in DC maturation. It also produces NK cell mediated 
ADCC and CDC which further enhances its tumoricidal activity. Currently, cetuximab is being tested in a phase II clinical trial in combination with a pancreatic cancer vaccine. ${ }^{26}$ Cytokines such as Interleukin 2 (IL-2) and Interferons (IFNs) and Interleukin-12 (IL-12) are also characterized as passive immunotherapy. IL-2 is produced by activated $\mathrm{T}$ cells. It does not primarily act on cancer cells; rather it acts as an adjuvant and stimulates immune reactions. ${ }^{5}$ IL-2 therapy has been found to be effective in melanoma and metastatic renal cell carcinoma. But it is associated with a side effect known as vascular leak syndrome..$^{27}$ IFNs are inflammatory cytokines that are produced by the body in response to certain infections. It has been shown that IFN- $\alpha$ inhibits proliferation of tumor cells and also as antiangiogenic activity against leukemia and lymphoma. It is used as an adjuvant therapy and facilitates activation of immune cells. ${ }^{9,28}$ IL-12 is an interleukin that is involved in differentiation of naïve $\mathrm{T}$ cells into T-helper Th1 or Th2 cells. It activates CTLs and NK cells, and supports the growth and function of T cells. Combination of HER2 $\mathrm{mAb}$ with systemic IL-12 has shown to decrease tumor progression and increase tumor necrosis as compared to treatments given individually. Recently, a phase I clinical trial, using combination of IL-2 with Herceptin and paclitaxel has shown increased production of IFNs. ${ }^{26}$ One obvious drawback with the use of mAbs in humans would be that the immune system may recognize these mAbs as foreign and eventually be destroyed by the body's immune system. But over the years, researchers have surmounted this obstacle by designing "chimeric" or humanized antibodies achieved by replacing some parts of the mouse antibody proteins with human proteins. ${ }^{9,21}$ Additional limitations of mAb therapy are listed in the (Table 1).

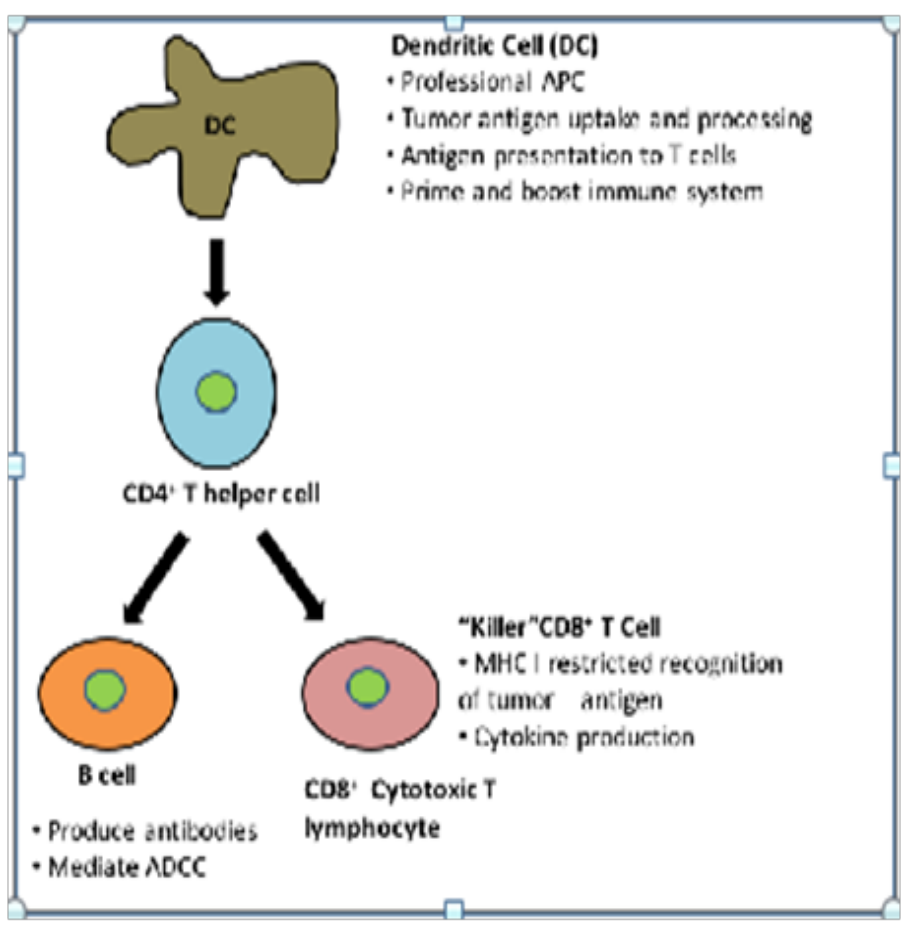

Figure I Key players of the Immune system.

\section{Active immunotherapy}

"Cancer vaccines" are considered as active immunotherapy as they boost the body's immune system to defend against cancer. Recently, active immunotherapy in the form of cancer vaccines has indicated some encouraging and promising results in many clinical trials. ${ }^{4}$ Active immunotherapy may be divided into two sub-categories, prophylactic or therapeutic. Prophylactic Cancer Vaccines are aimed to prevent cancer in patients who may be at high risk of developing cancer due to genetic predisposition or environmental factors. These function as "traditional vaccines" like flu vaccine. Prophylactic cancer vaccines target infections that may lead to development of cancer. The US FDA has approved two vaccines, namely- Gardesil and Cervarix, against Human Papilloma Virus (HPV) which is responsible for 70percent of cases of cervical cancer. ${ }^{8}$ Gardasil utilizes HPV antigens as proteins. These proteins are processed in the laboratory to synthesize four different types of 'virus like particles' or VLPs which are combined to form a cocktail that is effective against HPV infections of type 6,11, 16 and 18. This is superior to traditional vaccines consisting of weak or inactivated whole microbes which may sometimes be infectious. ${ }^{4}$ Similarly, Cervarix is composed of proteins from HPV type 16 and 18. US FDA has also approved vaccine against Hepatitis B virus (HBV) infection which is known to cause liver cancer. In all cases mentioned above the causative infectious agent is known. But the formulation of therapeutic vaccines in other types of cancer wherein the etiology is unclear would be a challenging task for future scientists. ${ }^{8}$ Therapeutic vaccines are intended to treat an existing tumor. Once cancer is diagnosed, a solid accessible tumor may be removed by surgery, chemotherapy or radiation therapy. After surgery the patient is vaccinated to develop a specific immune response to kill residual cancer cells and thus prevent relapse. ${ }^{29,30}$ Examples of therapeutic vaccines are listed in (Table 2). DC based vaccines have become a very popular form of cancer vaccine therapy considered relatively safe for use in humans. Two approaches may be used to design such vaccines. ${ }^{13,37}$ Ex vivo and in vivo which are outlined in (Table 3). Unfortunately, initial attempts to design cancer vaccines were not very successful as the actual mechanism of immunization was unclear. Now that the function of DCs in generating a potent $\mathrm{T}$ cell response is known, many clinical trials are exploiting this concept. DC based vaccines are being tested for a variety of cancers such as prostate, colorectal, kidney, breast cancer, melanoma, leukemia, lymphoma and other tumor malignancies. ${ }^{38,39}$ Cancer vaccines are designed to target cancer cells with the help of the immune system and so the success of cancer vaccines largely depends upon a well-functioning immune system of the individual's immune system receiving the vaccine. Factors affecting the immune system include: age ${ }^{40}$ immune suppression due to tumor microenvironment ${ }^{41}$ substances secreted by tumor, or immunosuppressive drugs like glucocorticoids, analgesics or anesthetics. ${ }^{42}$ Nutritional supplements may enhance the function of immune cells that play a key role in cancer immunotherapy. Previous studies in cancer patients indicate that nutritional supplements improve immune system functioning that may be adversely affected due to existing tumor or surgery. ${ }^{11}$ Despite tremendous research that is currently ongoing in the field of cancer vaccines, major hurdles still impede the success of cancer vaccines as listed in Table 4. Several new approaches are being investigated, including the use of co-stimulatory molecules, multiple dosing, using combinations of tumor antigens, etc., to overcome the above-mentioned limitations. However, not much effort is being focused on vaccine formulation and delivery routes. The formulation and route of delivery of the vaccine is also an important parameter in targeted systems which needs to be addressed soon and would be an integral part in the development of cancer immunotherapy-based interventions. 


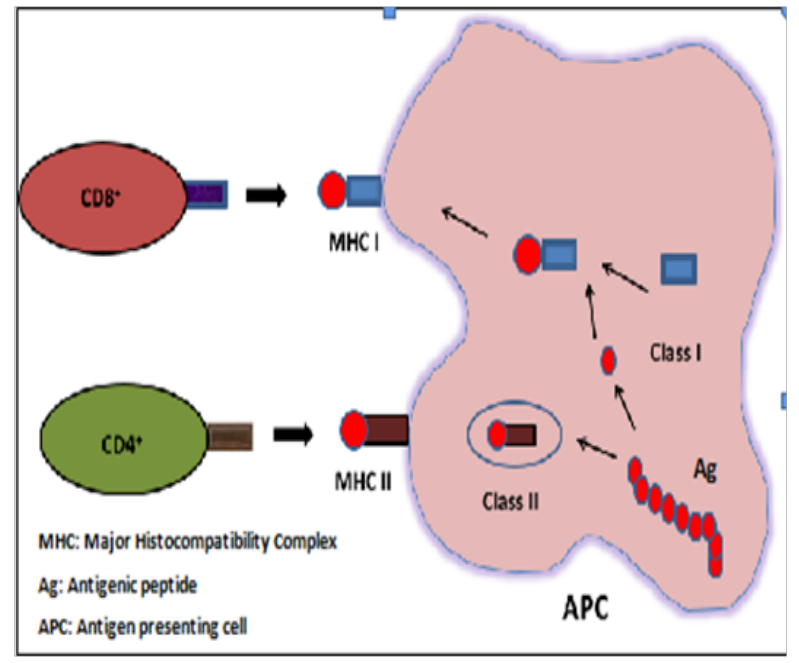

Figure 2 Activation of T cells via APCs.

Table I Limitations of monoclonal antibodies (mAb)

I. Change in expression level of target antigen due to mutations.

2. Use restricted to a subgroup of patients expressing the unique antigen.

3. Difficult to get a significant therapeutic response avoiding autoimmunity.

4. Tumor microenvironment is particularly immunosuppressive limiting mAb success.

Table 2 Therapeutic cancer vaccines

\begin{tabular}{ll}
\hline Whole cell based vaccines & $\begin{array}{l}\text { Synthetic protein antigen } \\
\text { vaccines }\end{array}$ \\
\hline $\begin{array}{l}\text { This approach uses inactivated whole } \\
\text { tumor cells that display a variety of } \\
\text { known or unknown oncogenic antigens } \\
\text { on their surface against which the body's } \\
\text { immune system develops an antitumor } \\
\text { response. }\end{array}$ & $\begin{array}{l}\text { These are synthetically } \\
\text { produced tumor specific } \\
\text { antigens that can generate } \\
\text { immune response in body } \\
\text { against cancer cells displaying } \\
\text { this antigen. }\end{array}$ \\
\hline
\end{tabular}

This approach may overcome the problem of identifying specific tumor antigen, majority of which are unknown till today but is still associated with the risk of autoimmunity.

Whole cell vaccines may be autologous or allogenic:

Autologous: If the tumor cells are obtained from patient itself then it is known as an autologous or self-tumor cell vaccine. ${ }^{31}$

Allogenic: if tumor cells are obtained from another individual having the same cancer then it may be called as an allogenic or donor vaccine. ${ }^{32}$

Dendritic Cell (DC) based vaccines: These may be either ex vivo or in vivo and are explained in detail in the next section.

This therapy is will produce a more tumor specific immune response compared to whole

Often combined with adjuvants that help to further enhance immune response. ${ }^{33}$ Examples of synthetic protein antigen based vaccines include:

Melanoma: MART-I, tyrosinase, gP-100. ${ }^{34}$

Breast and ovarian cancer: Sialyl-Tn (STn) ${ }^{35}$

Pancreatic, Lung,

Colorectal, Breast, and Ovarian Cancers:
Table 3 Dendritic cell based vaccines

Ex Vivo In Vivo

In this approach, DCs may be loaded with antigen by culturing DCs obtained from patients with a tumor specific antigen and an adjuvant (ex vivo) that will induce DC maturation. These cells are injected back into the patient.

This method involves ex vivo culture of the DCs in the laboratory and thus may have higher risk of endotoxin contamination.

\section{Cultured DCs should not lose migratory capacity to lymph nodes.}

Does not require targeting of DCs.

May be laborious and time consuming.

DCs may be induced to take up tumor antigen in vivo. Antitumor responses may take time to build but responses may be robust and long lasting.

Since DCs are induced in vivo to take up the tumor antigen considerable lesser risk of endotoxin contamination.

No risk of loss of migratory capacity for in vivo based vaccines.

This approach will require optimum in vivo targeting of DCs.

Relatively less laborious and time consuming.

Table 4 Limitations of cancer vaccines

Variable Antigen expression: Although a variety of tumor associated antigens have been identified, these antigenic peptides still differ in their ability to generate an effective immune response. I. Lately, tumor specific antigens have been discovered which further facilitate a tumor specific response, sparing the healthy cells and ruling out the possibility of autoimmunity. ${ }^{4,43}$

Low immunogenicity: Often cancer vaccines are unable to generate a strong immune response. This problem may be solved by 2. incorporation of "immune boosters" such as IL-2 and Granulocytemacrophage colony stimulating factor (GM-CSF) which act as costimulatory molecules and facilitate immune reactions. ${ }^{43}$

Tumor microenvironment: Even if an immune response is

3. generated, the tumor microenvironment may neutralize it. So some strategies must be employed to mitigate the immune suppressive nature of the tumor microenvironment cell approach. Carcinoembryonic antigen (CEA). ${ }^{36}$
Loss of efficacy: Vaccine may render the desired response initially,

4. but over the time the immune response may diminish which might lead to relapse.

Chemotherapy remains the backbone of cancer therapy. An array of oncogenic signaling pathways have been identified and targeted as points of intervention for cancer therapy. A multitude of inhibitors of such molecular pathways are available commercially or are under clinical investigation. ${ }^{26}$ However, in the past decade researchers, immunologists and oncologists have also focused on the role of the immune system to combat cancer via immunotherapy-based cancer vaccines. As stated earlier, like several other conventional therapies, cancer vaccines also have limitations. Several studies have shown the potential of using immunotherapy as an efficient adjuvant treatment modality along with chemotherapy. ${ }^{26}$ Thus, the future of cancer therapy lies in a combinatorial approach. Targeted chemotherapy and immunotherapy shows great promise to be the foundation of a new era of cancer therapeutics.

\section{Conclusion}




\section{Acknowledgements}

None.

\section{Conflict of interest}

Author declares that there is no conflict of interest.

\section{References}

1. Siegel R, Naishadham D, Jemal A. Cancer statistics for hispanics/latinos, 2012. CA Cancer J Clin. 2012;62(5):283-298.

2. Jabir NR, Tabrez S, Ashraf GM, et al. Nanotechnology-based approaches in anticancer research. Int J Nanomedicine. 2012;7:4391-4408.

3. Subbiah R, Veerapandian M, Yun KS. Nanoparticles: Functionalization and multifunctional applications in biomedical sciences. Curr Med Chem. 2010;17(36):4559-4577.

4. Types of biological cancer immunotherapy. 2015.

5. Rosenberg SA, Yang JC, Restifo NP. Cancer immunotherapy: Moving beyond current vaccines. Nat Med. 2004;10(9):909-915.

6. Brodsky FM, Guagliardi LE. The cell biology of antigen processing and presentation. Annu Rev Immunol. 1991;9(1):707-744.

7. Luckheeram RV, Zhou R, Verma AD, et al. CD4< sup. Clinical and developmental immunology. 2012

8. Cancer topics, factsheet. Therapy, cancer vaccines. National Cancer Institute, USA; 2015.

9. Cancer treatment, side effects, treatment types, immunotherapy, immunotherapy cancer vaccines. Cancer immunotherapy. 2015.

10. Parham P. Putting a face to MHC restriction. JImmunol. 2005;174(1):3-5

11. Baines J, Celis E. Immune-mediated tumor regression induced by CpG-containing oligodeoxynucleotides. Clin Cancer Res. 2003;9(7):2693-2700.

12. Ma J, Liu L, Che G, et al. The M1 form of tumor-associated macrophages in non-small cell lung cancer is positively associated with survival time. BMC Cancer. 2010;10:112.

13. Xu H, Cao X. Dendritic cell vaccines in cancer immunotherapy: From biology to translational medicine. Front Med. 2011;5(4):323-332.

14. Palucka K, Banchereau J. Cancer immunotherapy via dendritic cells. Nat Rev Cancer. 2012;12(4):265-277.

15. McCarthy EF. The toxins of william B. coley and the treatment of bone and soft-tissue sarcomas. Iowa Orthop J. 2006;26:154-158.

16. Richardson MA, Ramirez T, Russell NC, et al. Coley toxins immunotherapy: A retrospective review. Altern Ther Health Med. 1999;5(3):42-47.

17. Snook AE, Waldman SA. Advances in cancer immunotherapy. Discov Med. 2013;15(81):120-125.

18. Rosenberg SA. Cell transfer immunotherapy for metastatic solid cancer-what clinicians need to know. Nat Rev Clin Oncol. 2011;8(10):577-585.

19. Knuth A, Wölfel T, zum Büschenfelde KM. Cellular and humoral immune responses against cancer: Implications for cancer vaccines. Curr Opin Immunol. 1991;3(5):659-664

20. Elgert KD. Immunology: Understanding the immune system. USA: John Wiley \& Sons; 2009.

21. Conniot J, Silva JM, Fernandes JG, et al. Cancer immunotherapy: Nanodelivery approaches for immune cell targeting and tracking. Front Chem. 2014;2:105.
22. Junker U, Knoefel B, Nuske K, et al. Transforming growth factor beta 1 is significantly elevated in plasma of patients suffering from renal cell carcinoma. Cytokine. 1996;8(10):794-798.

23. Noguera R, Nieto OA, Tadeo I, et al. Extracellular matrix, biotensegrity and tumor microenvironment. an update and overview. Histol Histopathol. 2012;27(6):693-705

24. Igney FH, Krammer PH. Immune escape of tumors: Apoptosis resistance and tumor counterattack. J Leukoc Biol. 2002;71(6):907-920.

25. Scott AM, Wolchok JD, Old LJ. Antibody therapy of cancer. Nature Reviews Cancer. 2012;12(4):278-287.

26. Vanneman M, Dranoff G. Combining immunotherapy and targeted therapies in cancer treatment. Nat Rev Cancer. 2012;12(4):237-251.

27. Baluna R, Vitetta ES. Vascular leak syndrome: A side effect of immunotherapy. Immunopharmacology. 1997;37(2):117-132.

28. Guilhot F, Roy L, Guilhot J, et al. Interferon therapy in chronic myelogenous leukemia. Hematol Oncol Clin North Am. 2004;18(3):585-603.

29. Hellstrom KE, Hellstrom I. Novel approaches to therapeutic cancer vaccines. Expert Rev Vaccines. 2003;2(4):517-32.

30. Kufe DW, Pollock RE, Weichselbaum RR, et al. Holland-frei cancer medicine. 6th ed. USA: BC Decker; 2006.

31. Lahn M, Köhler G, Schmoor C, et al. Processing of tumor tissues for vaccination with autologous tumor cells. Eur Surg Res. 1997;29(4):292-302.

32. Chan AD, Morton DL. Active immunotherapy with allogeneic tumor cell vaccines: Present status. Semin Oncol. 1998;25(6):611-622.

33. Schulz M, Zinkernagel RM, Hengartner H. Peptide-induced antiviral protection by cytotoxic T cells. Proc Natl Acad Sci U SA. 1991;88(3):991-993.

34. Jäger E, Ringhoffer M, Karbach J, et al. Inverse relationship of melanocyte differentiation antigen expression in melanoma tissues and CD8 cytotoxic-T-cell responses: Evidence for immunoselection of antigenloss variants in vivo. Int J Cancer. 1996;66(4):470-476.

35. Holmberg LA, Sandmaier BM. Vaccination with theratope $($ STn-KLH) as treatment for breast cancer. Expert Rev Vaccines. 2004;3(6):655-63.

36. Marshall J. Carcinoembryonic antigen-based vaccines. Semin Oncol. 2003;30(3 Suppl 8):30-36.

37. Palucka K, Banchereau J, Mellman I. Designing vaccines based on biology of human dendritic cell subsets. Immunity. 2010;33(4):464-478.

38. Wiemann B, Starnes CO. Coley's toxins, tumor necrosis factor and cancer research: A historical perspective. Pharmacol Ther. 1994;64(3):529-564.

39. Mellman I, Coukos G, Dranoff G. Cancer immunotherapy comes of age Nature. 2011;480(7378):480-489.

40. Holmes FF, Wilson J, Blesch KS, et al. Biology of cancer and aging Cancer. 1991;68(S11):2525-2526.

41. De Cecco L, Berardi M, Sommariva M, et al. Increased sensitivity to chemotherapy induced by CpG-ODN treatment is mediated by microRNA modulation. PloS one. 2013;8(3):e58849.

42. Vallejo R, Hord ED, Barna SA, et al. Perioperative immunosuppression in cancer patients. J Environ Pathol Toxicol Oncol. 2003;22(2):139-146.

43. Fioretti D, Iurescia S, Fazio VM, et al. DNA vaccines: Developing new strategies against cancer. Journal of Biomedicine and Biotechnology. 2010;2010:1-16. 